\title{
Dasatinib worsens the effect of cetuximab in combination with fractionated radiotherapy in FaDu- and A431-derived xenografted
} tumours

\author{
M Baro ${ }^{1}$, L I de Llobet ${ }^{1}$, A Figueras ${ }^{1}$, I Skvortsova ${ }^{2}$, R Mesia ${ }^{3}$ and J Balart ${ }^{\star}, 1,4$ \\ ${ }^{1}$ Laboratory of Translational Research, Catalan Institute of Oncology, Avda. Gran de L'Hospitalet 199-203, 08907 L'Hospitalet de \\ Llobregat, Spain; ${ }^{2}$ Department of Therapeutic Radiology and Oncology, Innsbruck Medical University, Anichstrasse 35, 6020 \\ Innsbruck, Austria; ${ }^{3}$ Department of Medical Oncology, Catalan Institute of Oncology, Avda. Gran de L'Hospitalet 199-203, 08907 \\ L'Hospitalet de Llobregat, Spain and ${ }^{4}$ Department of Radiation Oncology, Hospital de la Santa Creu i Sant Pau, Sant Antoni Maria \\ Claret 167, 08025 Barcelona, Spain
}

Background: Cetuximab is often combined with radiotherapy in advanced SCCHN. Alternative routes bypassing inhibition of EGFR with cetuximab may overshadow the efficacy of this combination. We undertook this study to investigate a possible role of dasatinib in this scenario.

Methods: The SCC5, SCC25, SCC29, FaDu and A431 cell lines were assessed in vitro for cell proliferation under cetuximab and dasatinib treatments. In FaDu and A431 cells, dasatinib plus cetuximab resulted in higher proliferation than cetuximab alone. Then, FaDu and A431 cells were implanted into subcutaneous tissue of athymic mice that were irradiated with 30 Gy in 10 fractions over 2 weeks, and treated with cetuximab and dasatinib. Tumour growth, DNA synthesis and angiogenesis were determined. The EGFR, RAS-GTP activity, phosphorylated AKT, ERK1/2, SRC protein levels and VEGF secretion were determined in vitro.

Results: The addition of dasatinib to cetuximab and radiotherapy increased tumour growth, DNA synthesis and angiogenesis that were associated with RAS, AKT and ERK1/2 activation, and SRC inhibition in FaDu and A431 cells.

Conclusions: In xenografts derived from these two cell lines, dasatinib did not improve the efficacy of cetuximab combined with radiotherapy. On the contrary, it worsened tumour control achieved by the combination of these two treatments.

\begin{abstract}
Aberrant epidermal growth factor receptor (EGFR) signalling drives oncogenesis in many carcinomas, including squamous cell carcinoma of the head and neck (SCCHN), non-small-cell lung cancer (NSCLC) and colorectal cancer (CRC). Epidermal growth factor receptor is typically activated by EGF, TGF- $\alpha$ and amphiregulin ligands that are abnormally produced by cancer cells and tumour-associated stromal cells (Wyckoff et al, 2004). In other instances, amplification or punctual mutations in the EGFR gene will originate an excessive function of the EGFR. Moreover, radiation-induced activation of EGFR occurs in a
\end{abstract}

ligand-independent manner with doses usually applied in radiotherapy (1-5 Gy) (Schmidt-Ullrich et al, 1997). Irrespective of the mechanism of activation, EGFR emits growth signals that are conveyed by proteins integrated into the RAS/ERK1/2 (extracellular-signal-regulated kinases 1 and 2) and phosphatidylinositol 3-kinase/AKT (protein kinase B) pathways (Jorissen et al, 2003; Sordella et al, 2004). Epidermal growth factor receptor is also translocated into the cell nucleus, where its tyrosine kinase (TK) activity is fully preserved (Dittmann et al, 2005, 2010), and able to interact with transcriptional factors and nuclear proteins to 
promote oncogenesis (Lin et al, 2001). Epidermal growth factor receptor confers sustained cell proliferation and increased survival on cancer cells, tumour invasion and angiogenesis (Hunter, 1997; Hanahan and Weinberg, 2000; Malumbres and Barbacid, 2003; Hanahan and Weinberg, 2011), and its overexpression is a major origin of radiation resistance and a relevant cause of local relapses after radiotherapy (Liang et al, 2003).

The blockage of EGFR by the monoclonal antibody cetuximab (C225) in combination with radiotherapy has been shown to improve survival and local control in locally advanced head and neck cancers (Bonner et al, 2006). In this setting, however, major responses are limited as a consequence of intrinsic or acquired cellular resistance to cetuximab that may be based on the robustness of signalling networking that grants alternative routes to avoid EGFR blockage (Amit et al, 2007). One route of resistance to EGFR blockage is the cross-talking with the oncoprotein SRC. The SRC interacts with EGFR to phosphorylate it at tyrosine 845 (Tice et al, 1999), a necessary step for full activity of EGFR (Sato et al, 2003). The SRC kinases can be activated by reactive oxygen species such as those induced by ionising radiation (Giannoni et al, 2005). It also mediates the internalisation of EGFR into caveolae to shuttle EGFR to the cell nucleus (Dittmann et al, 2008). In addition, SRC-family kinases occupy a central position within the cell signal networking, interact with most oncogenic signalling pathways (Elsberger et al, 2010) and participate in the differentiation, adhesion and motility of cells and angiogenesis (Summy and Gallick, 2006). This central role makes SRC a potential target in cancer treatment, independently of whether SRC suffers or not an activating mutation. In the current study, we attempted to potentiate cell sensitivity to cetuximab by using dasatinib, an FDA-approved drug that works as ATP-competitive TK inhibitor that largely targets cytosolic SRC (Lombardo et al, 2004).

The major outcome of our study, however, was that dasatinib worsened the effect in combination with cetuximab or cetuximab and radiation therapy in $\mathrm{FaDu}$ and $\mathrm{A} 431$ cell-derived tumours, inducing a paradoxical increase of tumour growth, higher DNA synthesis and elevated angiogenesis.

\section{MATERIALS AND METHODS}

Cell lines and pharmacological inhibitors. In this study, we used the SCCHN SCC5, SSC25, SCC29 and FaDu cell lines. The FaDu and SSC25 cell lines were obtained from the American Type Culture Collection (ATCC, Manassas, VA, USA). The SCCHN SCC5 and SCC29 cell lines were a kind gift from R Alemany (Institut Català d'Oncologia, Barcelona, Spain). We also used the human cell line A431 derived from a vulvar squamous primary carcinoma (ATCC). The cell line SCC29 expresses relatively low levels of EGFR (Erjala et al, 2006). The cell lines SCC5 and SCC25 express moderate levels of EGFR (Nowsheen et al, 2011; Wu et al, 2011; Lin et al, 2012). The $\mathrm{FaDu}$ and A431 cell lines overexpress EGFR, although FaDu to a lesser degree than A431 (Song et al, 2009). The levels of total EGFR in these cells were assessed confirming published data (Supplementary Figure 1). We also verified that Kirsten-ras gene (K-RAS) of these cell lines was wild type according to the DNA PCR analysis of codons 12 and 13 of exon 2 of K-RAS gene (Supplementary Table 1). The cells were cultured under standard conditions according to ATCC recommendations, and they were kept in culture not more than 6 months after resuscitation from original stocks. Mycoplasma cell culture contamination was routinely checked and ruled out by PCR.

Commercially available monoclonal antibody anti-EGFR cetuximab (Merck KGaA, Darmstadt, Germany) and the SRC kinase inhibitor dasatinib (BMS-354825; LC Laboratories, Woburn, MA, USA) were used to treat cell cultures and mice. Dasatinib was diluted in DMSO (Sigma, St Louis, MO, USA) for in vitro experiments, and in 1,2-propanediol (Sigma) in water $1: 1(\mathrm{v} / \mathrm{v})$ for the treatment of mice. Cell cultures were also treated with the ATP-competitive TK SRC inhibitor PP2 (AG1879) and EGFR inhibitor AG1478 (Calbiochem, San Diego, CA, USA).

Xenografted tumours and in vivo treatments. The effect of radiotherapy, cetuximab and dasatinib was evaluated in mice bearing xenografted tumours. Female athymic Swiss nu/nu mice, 6-8 weeks old, were purchased from Harlan (Gannat, France). Tumours were established by subcutaneous injection of $\mathrm{FaDu}$ or A431 cells into hind limb. Radiotherapy consisted of $30 \mathrm{~Gy}$ in 10 fractions. Details of the radiotherapy technique have been published elsewhere (Baro et al, 2012). Cetuximab and dasatinib were administered concomitantly with radiotherapy (treatment schedules are described in the Figure 2 legend). Equivalent mock treatments were performed as controls. Tumour size was calculated according to the formula $\pi / 6 \times($ large diameter $) \times(\text { small diameter })^{2}$. Mice were killed when the tumour volume reached $1000 \mathrm{~mm}^{3}$, when they suffered moderate to severe toxicities, when significant differences between groups were observed or when less of three animals were followed for tumour volume assessment. All experimental procedures were approved in accordance with our own institutional guidelines for animal care and ethics and guidelines for the welfare and use of animals in cancer research (Workman et al, 2010).

BrdU incorporation assay. Tumour DNA synthesis was estimated by immunofluorescence staining of the 5-bromo2 -deoxyuridine (BrdU) (Invitrogen, Carlsbad, CA, USA). At $1 \mathrm{~h}$ before tumour sampling, BrdU was intraperitoneally injected. Briefly, cryostat sections (3- $\mu \mathrm{m}$ thick) of xenografted tumours were embedded in OCT compound (Sakura Finetek Europe, Zoeterwoudl, The Netherlands). Fixed samples were washed $(0.1 \%$ Triton), incubated for $20 \mathrm{~min}$ in $\mathrm{HCl} 2 \mathrm{~N}$ and washed with $0.1 \mathrm{M}$ borate buffer ( $\mathrm{pH} \mathrm{8)}$. After protein blocking, slices were incubated overnight with rat anti-BrdU primary (AbD Serotec, Oxford, UK), and for $1 \mathrm{~h}$ with Alexa Fluor 488-conjugated rabbit anti-rat secondary antibody (Invitrogen). Then, slices were mounted using Vectashield (Vector Laboratories Inc., Burlingame, CA, USA) mounting medium with 4,6-diamidino-2-phenylindole (DAPI). Fluorescence images were captured using a Nikon Eclipse 80i epifluorescence microscope (Nikon Instruments, Kanagawa, Japan) and analysed using Nis-Elements, Basic Research (Nikon) software. The BrdU index was calculated as the ratio between green fluorescence (BrdU incorporated to S-phase nuclei) and blue fluorescence (DAPI incorporated to nuclei).

CD31/PECAM immunostaining and VEGF determination. Angiogenesis was assessed by determination of the mouse platelet and endothelial cell adhesion molecule antigen (CD31/PECAM). A standard method, described elsewhere, was applied (Pueyo et al, 2010). Microvessel density (MVD) was determined under light microscopy following the criteria described by Weidner et al (1991). Vascular endothelial growth factor (VEGF) was determined in supernatants of cell cultures. The FaDu or A431 cells were plated and allowed to grow for $24 \mathrm{~h}$. Cells were treated in fetal bovine serum (FBS)-free medium with radiotherapy alone, or radiotherapy combined with cetuximab alone or with both cetuximab and dasatinib. Vascular endothelial growth factor was evaluated by ELISA assay (R\&D Systems Inc., Minneapolis, MN, USA) at 0, 24, and $48 \mathrm{~h}$ as previously reported (Pueyo et al, 2010).

Western blot analysis and RAS-GTP assay. Cells were treated with cetuximab, dasatinib or both in FBS-free medium. Following treatment, western blot analyses were performed as previously described (Pueyo et al, 2010). We used the following primary antibodies: rabbit anti-pEGFR-Y845 $(1: 1000)$, rabbit anti-pSRCY418 (1:1000), mouse anti-pERK1/2 $(1: 1000)$ and mouse anti$\alpha$-tubulin (1:5000; all from Sigma) and rabbit anti-pAKT-S473 $(1: 1000)$ antibody from Cell Signalling (Danvers, MA, USA). The nitrocellulose-bound primary antibodies were incubated for 
$1 \mathrm{~h}$ with anti-mouse IgG or anti-rabbit IgG horseradish peroxidaselinked antibody (GE Healthcare-Amersham Pharmacia, Buckinghamshire, UK), and were detected by the enhanced chemoluminescence staining ECL/ECL Plus (GE Healthcare).

To determine the activity of RAS, the RAS activation assay kit (Upstate Millipore, Temecula, CA, USA) was used. A total of $400 \mu \mathrm{g}$ protein from cell lysates was incubated with agaroseglutathione beads bound to glutathione-S-transferase (GST) fusion protein corresponding to the human RAS Binding Domain (RBD) of Raf-1. Then, agarose beads were collected by pulsing in a microcentrifuge and boiled in Laemmli buffer to obtain pull-down samples with the activated RAS. Then, RAS protein was assessed by western blot using a mouse anti-RAS clone10 antibody $(1: 1000)$ in GST pull-down and whole lysates (Millipore-Upstate, Billerica, MA, USA) and a goat anti-GST antibody $(1: 1000)$ or a mouse $\alpha$-tubulin antibody $(1: 5000)$ (GE Healthcare), respectively.

Statistical analysis. Results are expressed as mean \pm s.d. unless otherwise indicated. The Statistical Package for Social Sciences (SPSS, version 13.0, IBM, Madrid, Spain) was used for data analysis. Statistically significant differences in between-group comparisons were defined at a significance level of $P$-value $<0.05$ in the Mann-Whitney test.

\section{RESULTS}

Dasatinib worsens the effect of cetuximab on cell proliferation in FaDu and A431 cell lines. To explore whether dasatinib and cetuximab could interact in a synergistic manner, we first evaluated their effects in vitro in a group of four cell lines derived from SCCHN (SCC5, SCC25, SCC29 and FaDu), and in A431 cell line. We found that, as single treatments, both agents inhibited cell proliferation but with different efficacies (Figure 1A). Whereas treatment with dasatinib showed little activity against FaDu cells (Figure 1A), in the other three SCC cell lines a higher sensitivity to it was observed. Consistent with our results, it has been previously described that FaDu cells are relatively resistant to dasatinib (Lin et al, 2012). Similar to FaDu cell line, A431 cells were also poorly responsive to dasatinib (Figure 1A).

The addition of dasatinib to cetuximab resulted in a significant reduction of cell proliferation in all SCCHN (Figure 1A) compared with cetuximab alone with the exception of $\mathrm{FaDu}$ cell line. Unexpectedly, in FaDu cells, the combination of drugs resulted in a significant decrease of the effect of cetuximab alone (Figure 1A). Interestingly, in A431 cells - which were also poorly responsive to dasatinib alone - a lesser reduction of cell proliferation with the combination of the drugs compared with cetuximab alone was also observed (Figure 1A).

To further investigate cell proliferation, we examined possible dasatinib-induced variations in the phosphorylated levels of ERK1/2 proteins, proteins whose activation typically precedes cell cycle progression and mitogenesis induced by EGFR signalling. In SCC5 and SCC2 5 cells, EGF-stimulated levels of pERK1/2 were inhibited by the antibody cetuximab and followed by a higher inhibition in the presence of dasatinib (Figure $1 \mathrm{~B}$, lanes $\mathrm{E}$ and $\mathrm{CE}$ without dasatinib compared with lanes E and CE with dasatinib). In SCC29 cells, although treatment with cetuximab increased pERK1/2 levels
A

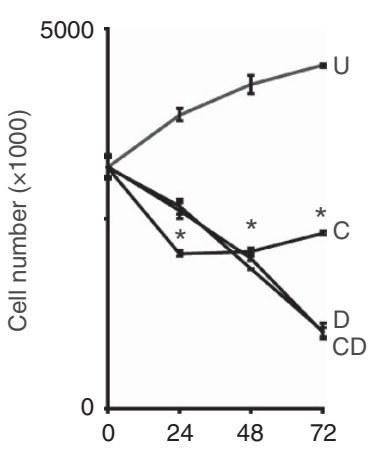

SCC25 cells

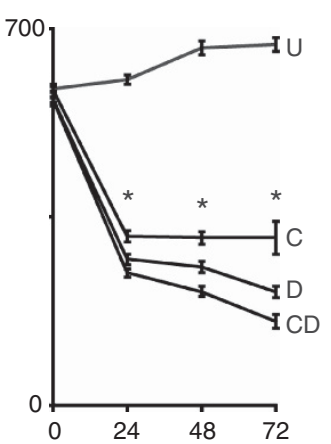

SCC29 cells

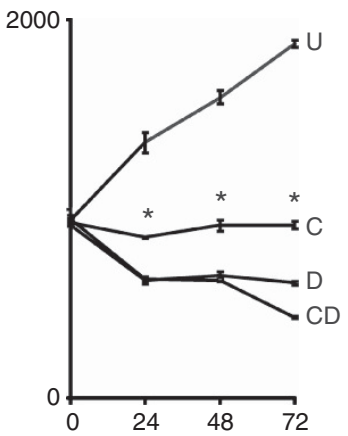

FaDu cells

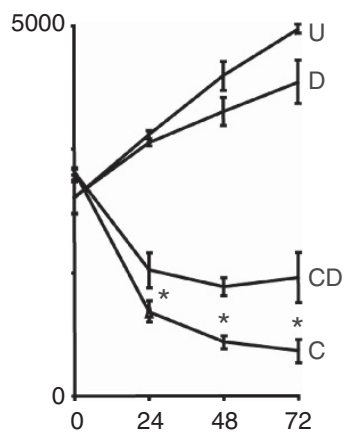

A431 cells

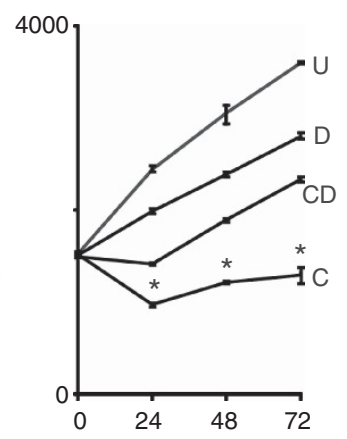

Hours from the start of treatment

B

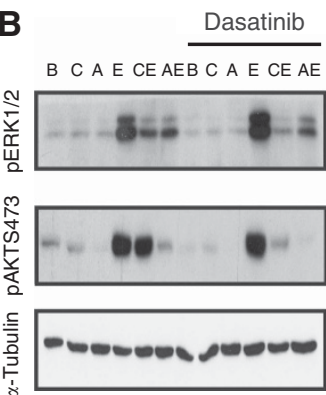

Dasatinib
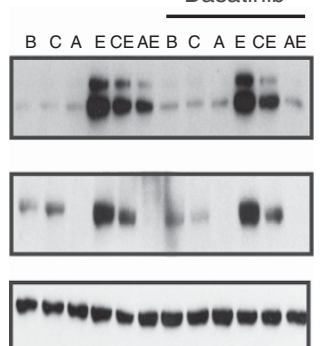
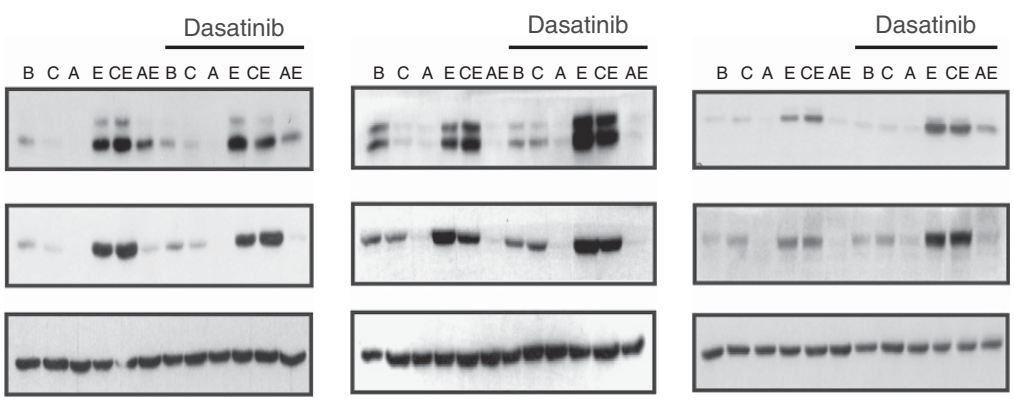

Figure 1. Differential effect of dasatinib on cell proliferation. The SCC5, SCC25, SCC29, FaDu and A431 cells were plated ( $\approx 12000$ cells per cm ${ }^{2}$ in $60 \mathrm{~mm}$ dishes) in complete medium and incubated for 5 days before drug treatment. For in vitro cell proliferation (A), cultures were treated with vehicles (U), $30 \mathrm{~nm}$ cetuximab (C), $150 \mathrm{~nm}$ dasatinib (D) or both at the same doses as monotherapy (CD) for 24, 48 and $72 \mathrm{~h}$. Cell proliferation from the start of treatments was assessed by counting the number of cells over the time course. Mean \pm s.e. values of two independent assays per duplicate and per cell line were calculated. ${ }^{*} P$-value $<0.05$ vs combined treatment with cetuximab and dasatinib (CD), (Mann-Whitney test).

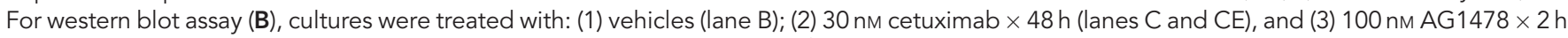
(lanes $A$ and $A E$ ). Before cell lysis, cultures were stimulated with $10 \mathrm{ng} \mathrm{ml}{ }^{-1} \mathrm{EGF}$ ligand $\times 10$ min (lanes $\mathrm{E}, \mathrm{CE}$ and $\mathrm{AE}$ ). In parallel, a set of cultures was additionally treated with $150 \mathrm{~nm}$ dasatinib $\times 48 \mathrm{~h}$. $\alpha$-Tubulin was used as a loading control. $\alpha$-Tubulin, pERK1/2 and pAKT were analysed by western blot in whole lysates. 
(occasional cetuximab-induced phosphorylation of ERK1/2 has been described elsewhere (Raben et al, 2005)), further treatment with dasatinib decreased pERK1/2. On the contrary, in FaDu and A431 cell lines the addition of dasatinib in the presence of EGF stimulus resulted in an increase in the phosphorylation of ERK1/2 proteins, irrespective of whether cetuximab was present or not (Figure $1 \mathrm{~B}$, lanes $\mathrm{E}$ and $\mathrm{CE}$ without dasatinib compared with lanes $\mathrm{E}$ and $\mathrm{CE}$ with dasatinib). The mitogenic AKT protein followed a similar pattern of phosphorylation as ERK1/2 proteins in SCC5 cells and showed less sensitivity to dasatinib in SCC25 and SCC29 cells (Figure 1B). In FaDu and A431 cells, DST induced elevated levels of phosphorylated AKT protein as well as ERK1/2 proteins. Western blot findings in Figure $1 \mathrm{~B}$ are compatible with the cell proliferation depicted in Figure 1A. It is noteworthy to point out that AG1478 inhibitor inhibited phosphorylation of ERK1/2 and AKT irrespective of whether or not the cells were treated with dasatinib. To summarise, these results indicate a dissimilar response to dasatinib: whereas in SCC cell lines dasatinib was associated with a decrease of cell proliferation and pERK1/2 levels, in $\mathrm{FaDu}$ and $\mathrm{A} 431$ cells dasatinib was associated with cell proliferation and an antagonistic increase in phosphorylated levels of ERK1/2 and AKT proteins.

Dasatinib worsens the effect of radiotherapy combined with cetuximab in FaDu and A431 xenografted tumours. To further evaluate a possible antagonistic interaction, we examined tumour growth after fractionated radiotherapy plus cetuximab with or without dasatinib in $\mathrm{FaDu}$ and A431 cells. Growing tumours were randomly assigned to receive treatments, each administered as a monotherapy as well as in combination. In FaDu-derived tumours, of the three agents, cetuximab had the highest antitumour effect, and in this case the mean tumour volume at day 35 was $646 \pm 367 \mathrm{~mm}^{3}$ comparable to the volume that tumours had at day 21 (14 days earlier) in untreated mice $\left(642 \pm 129 \mathrm{~mm}^{3}\right)$ (Supplementary Figure 2). In combination, radiotherapy and cetuximab caused the highest antitumour effect. At day 35, tumours had a mean volume of $432 \pm 90 \mathrm{~mm}^{3}$ that was approximately the mean volume at day 14 in untreated mice $\left(512 \pm 308 \mathrm{~mm}^{3}\right)$. The statistical analysis revealed significant differences $v s$ untreated tumours only at day $14(P$-value $=0.020)$, likely because of the high values in s.d. values. However, the analysis comparing radiation plus cetuximab to radiation alone resulted in a significant decrease in tumour growth in earlier time points and, importantly, differences were maintained during the follow-up (Supplementary Figure 2). Comparison between mice receiving radiotherapy plus cetuximab vs cetuximab alone or any other combination did not show significant differences. Intriguingly, the addition of dasatinib to radiotherapy or to cetuximab did not show an increased antitumour effect. We then evaluated whether the addition of dasatinib to the combination of radiotherapy and cetuximab could improve tumour control. We found, however, that dasatinib resulted in a significant loss of antitumour response (Figure 2). These results were consistent with Figure 1, and taken together they indicate that in $\mathrm{FaDu}$ cells dasatinib negatively influenced the tumour control induced by cetuximab, a sort of interaction that may be even spurred by radiation.

In A431-derived tumours, we found a response that recapitulated results in FaDu-derived tumours. Treatment with cetuximab alone or with radiotherapy plus cetuximab significantly inhibited tumour growth (tumour volume at day 21 for cetuximab and radiotherapy plus cetuximab treatments was $358 \pm 92 \mathrm{~mm}^{3}$ and $214 \pm 98 \mathrm{~mm}^{3}$, respectively, in contrast to significant higher values in untreated tumours; Supplementary Figure 2), whereas significant inhibition was not observed between untreated mice and radiotherapy- or dasatinib-treated mice, as single agents, or double treatment with cetuximab and dasatinib or radiotherapy and
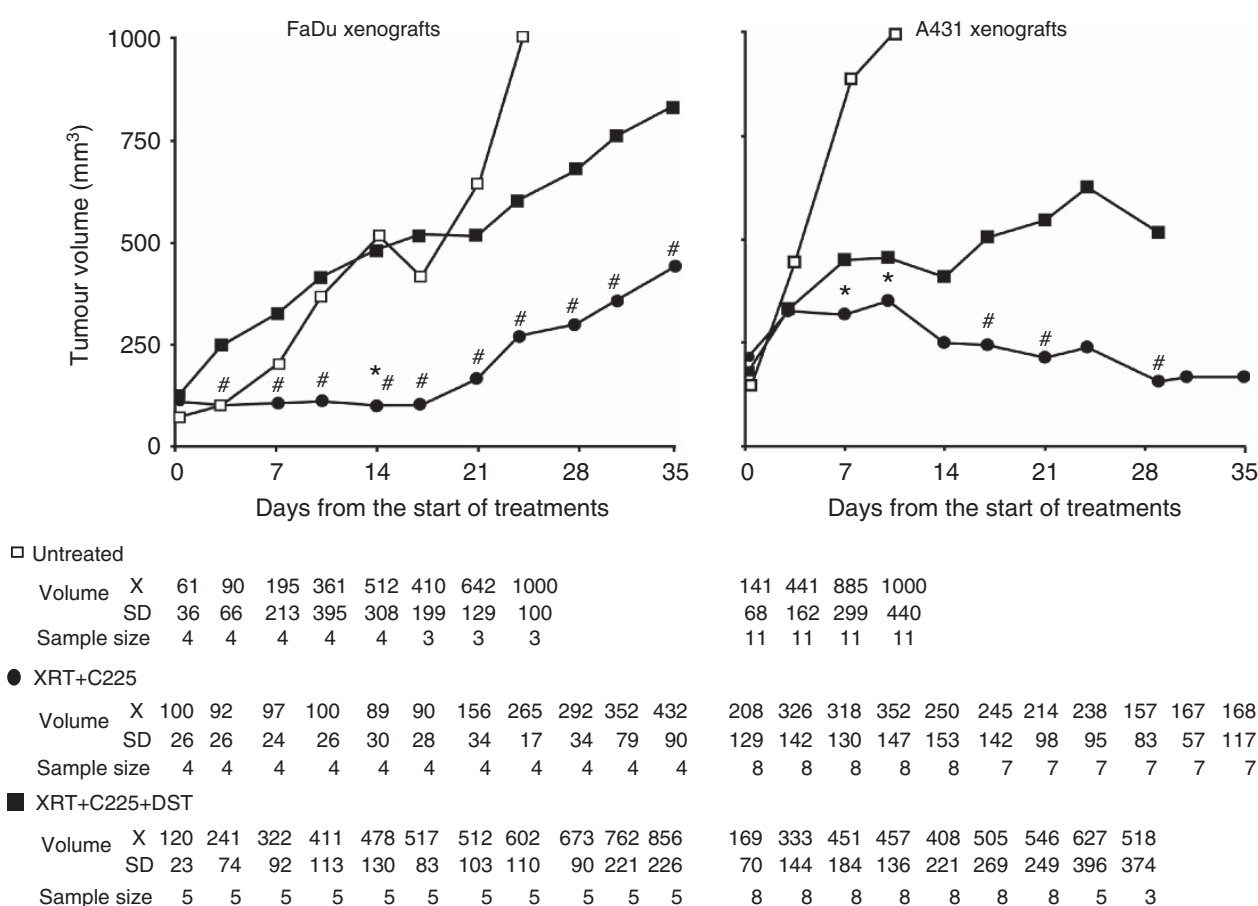

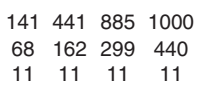

$\begin{array}{llll}11 & 11 & 11 & 11\end{array}$

$\begin{array}{rrrrrrrrrrr}208 & 326 & 318 & 352 & 250 & 245 & 214 & 238 & 157 & 167 & 168 \\ 129 & 142 & 130 & 147 & 153 & 142 & 98 & 95 & 83 & 57 & 117\end{array}$

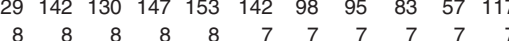

$\begin{array}{lllllllll}169 & 333 & 451 & 457 & 408 & 505 & 546 & 627 & 518\end{array}$ $\begin{array}{lllllllll}70 & 144 & 184 & 136 & 221 & 269 & 249 & 396 & 374\end{array}$

$\begin{array}{lllllllll}8 & 8 & 8 & 8 & 8 & 8 & 8 & 5 & 3\end{array}$

Figure 2. Dasatinib (DST) worsens the effect of radiotherapy (XRT) combined with cetuximab (C225) in FaDu and A431 xenografted tumours. The FaDu- and A431-derived tumours were established by subcutaneous injection of $1 \times 10^{6}$ cells into the right hind limb of athymic mice. Mice were randomly treated from day 0 to day 14 with: (1) XRT, 10 daily (except for Saturday and Sunday) consecutive localised doses of 3 Gy using $6 \mathrm{MV}$ X-ray; and (2) C225, $1 \mathrm{mg}$ per mice cetuximab intraperitoneally given $6 \mathrm{~h}$ before the first fraction of radiotherapy and $0.5 \mathrm{mg}$ per mice on days 4,8 and 11 at $2 \mathrm{~h}$ before radiotherapy. Numbers below the graphic indicate the values of tumour growth $(\mathrm{X}=$ mean volume) and remaining mice over time from the start of treatment that is represented on the $x$ axis of the graphic. ${ }^{*} P$-value $<0.05$ vs untreated, and ${ }^{\#} P$-value $<0.05$ vs $\mathrm{XRT}+\mathrm{C} 225+\mathrm{DST}$ (Mann-Whitney test). 
dasatinib. Consistent with previous findings (Figure 1), the addition of dasatinib to radiotherapy plus cetuximab was also associated with a significant decrease in tumour control (with $P$-values ranging from 0.025 to 0.048 ; Figure 2 ).

Dasatinib increases DNA synthesis and tumour-associated angiogenesis in $\mathrm{FaDu}$ and $\mathrm{A} 431$ xenografted tumours treated with radiotherapy and cetuximab. To investigate whether the dasatinib-induced tumour growth in $\mathrm{FaDu}$ and A431 cells could actually be related to an increase in cell proliferation, we determined the incorporation of BrdU, a DNA synthesis marker, in the xenografts. The number of BrdU-positive cells was higher in the tumours of mice treated with dasatinib. In FaDu-derived tumours, we observed that the BrdU/nucleus ratio was $38 \pm 6 \%$ in untreated mice, decreasing to $26 \pm 6 \%$ in mice that received radiotherapy and cetuximab $(P$-value $<0.001)$. However, this value increased to $42 \pm 6 \%$ in tumours treated with the triple treatment, a value that was significantly higher compared with the values in either untreated $(P$-value $=0.033)$ or double-treated mice $(P$-value $<0.001$; Figure 3A). In A431-derived tumours, these figures were $50 \pm 6 \%, 38 \pm 6 \%$ and $55 \pm 8 \%$, respectively, showing $P$-values similar to those found in $\mathrm{FaDu}$ tumours (Figure $3 \mathrm{~B}$ ), indicating that in both cell types dasatinib was associated with an increase in the synthesis of DNA and thus cell proliferation.

Given that tumour progression is often followed by neoangiogenesis, we decided to investigate whether the increased DNA synthesis was also associated with angiogenesis. We found that the presence of endothelial cells was slightly increased in tumours treated with dasatinib. In FaDu-derived tumours, MVD was $19 \pm 0$ in untreated tumours, and this value significantly decreased to $15 \pm 2$ in tumours treated with radiotherapy and cetuximab $(P$ value $=0.040)$. However, in mice treated with the triple treatment, MVD was $21 \pm 2$, similar to untreated mice $(P$-value $=0.182)$ and higher than in the double-treated mice group $(P$-value $=0.004$; Figure $3 \mathrm{C})$. In A431 tumours, the MVD was $11 \pm 2,10 \pm 1$ and $16 \pm 2$ in untreated, double-treated and triple-treated mice, respectively, with the differences in MVD between double- and triple-treated mice being significant $(P$-value $=0.009$; Figure 3D). Taken together, these findings are consistent with the notion that dasatinib was also involved in tumour-associated angiogenesis promotion.

Dasatinib increases secretion of VEGF in FaDu and A431 cell lines treated with radiotherapy and cetuximab. In light of the increased MVD observed in tumours treated with dasatinib, we hypothesised that VEGF secretion might augment in response to treatment with dasatinib. To test this, we treated $\mathrm{FaDu}$ and $\mathrm{A} 431$ cell cultures with radiotherapy, cetuximab and dasatinib. We found that levels of VEGF in supernatants of irradiated cultures increased in a time-dependent manner in both cell lines (Figure 4A and B). We also observed that at $48 \mathrm{~h}$, cetuximab inhibited the radiationinduced secretion of VEGF by a factor of 3 in both cell lines (from $9.7 \pm 5.2$ to $2.9 \pm 0.1$ for $\mathrm{FaDu}$ cells, $P$-value $=0.014$; and from $19.9 \pm 9.7$ to $5.8 \pm 4.0$ for A431 cells, $P$-value $=0.028$; Figure $4 \mathrm{~A}$ and $\mathrm{B}$, respectively), a result that was consistent with an earlier report from our group (Pueyo et al, 2010). However, the addition of dasatinib caused a significant increase in VEGF levels at $48 \mathrm{~h}$ in both types of cells (in agreement with elevated MVD observed in the tumours), counteracting cetuximab effect on radiation-induced secretion of VEGF by a factor of around 2 (from $2.9 \pm 0.1$ to $6.1 \pm 2.1$ for $\mathrm{FaDu}$ cells, $P$-value $=0.013$; and from $5.8 \pm 4.1$ to $11.7 \pm 1.6$ for A431 cells, $P$-value $=0.016$; Figure 4 ).

Dasatinib inhibits SRC tyrosine kinase in FaDu and A431 cells. In order to further explore the origin of the paradoxical elevation of pAKT and pERK kinases, we examined the modifications induced by dasatinib in EGFR-dependent cell signal transduction. First of all, we wanted to verify the effects of dasatinib on the activity of SRC in these cells. We found that dasatinib inhibited SRC in FaDu and A431 cells (Figure 5A), as evidenced by the low levels of phosphorylation at the activating site Y418 within the TK domain of the SRC protein (Figure 5A) (Roskoski, 2004; Luo et al, 2008). We also found that the phosphorylation of residue Y845 on EGFR, which is
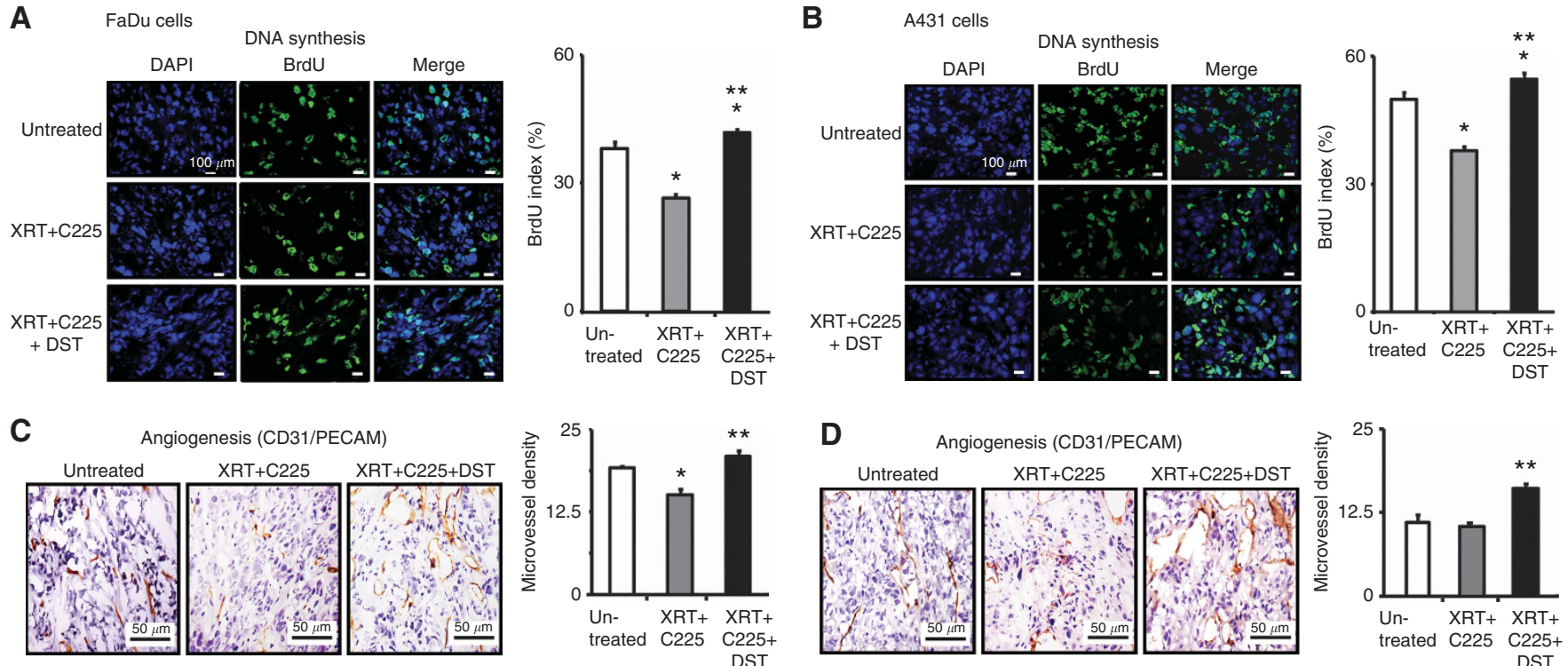

Figure 3. Dasatinib (DST) increases DNA synthesis and tumour-associated angiogenesis in FaDu and A431 xenografted tumours treated with cetuximab (C225) and radiotherapy (XRT). Xenografted tumours were treated in parallel as described in Figure 2 and sampled on day 4 from the start of treatment. (A) Representative images of immunofluorescence for BrdU incorporation in FaDu tumours and (B) A431 tumours. At $1 \mathrm{~h}$ before tumour sampling, BrdU at $50 \mathrm{mg} \mathrm{kg}^{-1}$ was injected intraperitoneally. Five tumours were evaluated per treatment condition with five random microscopic fields per tumour. (C) Representative images of standard immunohistochemistry for CD31/PECAM antigen in FaDu tumours and (D) A431 tumours. Microvessel density (MVD) was assessed using five independent tumours and five fields per tumour. Columns are means, bars are s.d. ${ }^{\star} P$-value $<0.05$ vs untreated tumours; ${ }^{\star \star} P$-value $<0.05$ vs XRT + C225 treatment (Mann-Whitney test). 

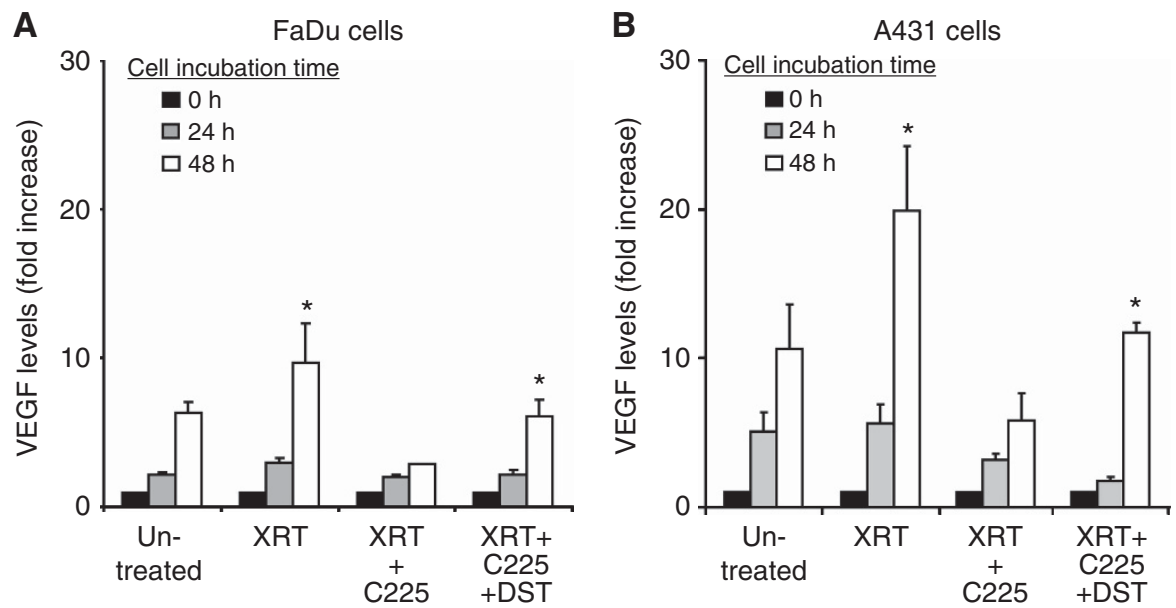

Figure 4. Dasatinib (DST) increases VEGF secretion in FaDu and A431 cell cultures treated with cetuximab (C225) and radiotherapy (XRT). (A) The FaDu cells and (B) A431 cells were plated ( $\approx 45000$ cells per $\mathrm{cm}^{2}$ in $60 \mathrm{~mm}$ dishes) and cultured for $24 \mathrm{~h}$ in complete medium and $24 \mathrm{~h}$ in FBS free medium. Then, cells were treated with: (1) a single dose of XRT (8 Gy), (2) XRT (8 Gy) + C225 (30 nM) or (3) XRT (8 Gy) + C225 (30 nm) + DST $(150 \mathrm{~nm})$. Supernatants were collected before treatments $(0 \mathrm{~h})$, and $24 \mathrm{~h}$ and $48 \mathrm{~h}$ later, the periods of time in which drug exposure was maintained. Vascular endothelial growth factor was determined by ELISA assay and normalised to cell number. Variations because of the time of incubation were reported as increments relative to time $0 \mathrm{~h}$. Two independent experiments for FaDu and three for A431 were performed, and each experiment was done per duplicate. Columns are means, bars are s.d. * $P$-value $<0.05$ vs XRT + C225 treatment (Mann-Whitney test).

A

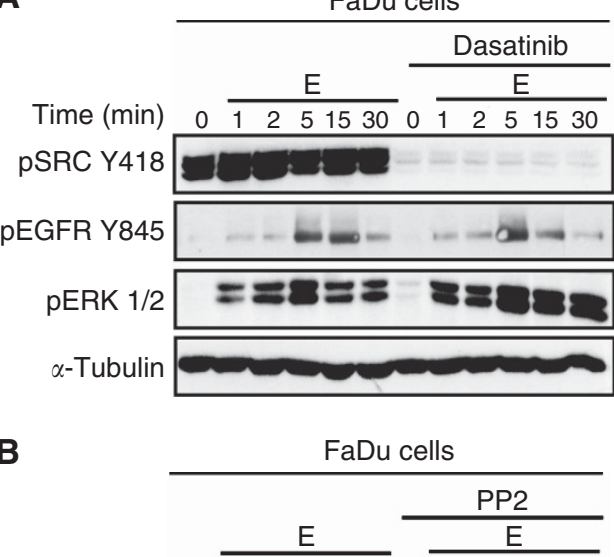

Time (min) $0 \overline{1251530} 0 \overline{1251530}$

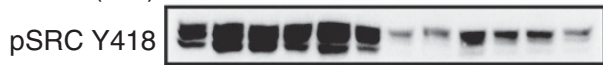

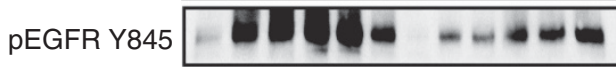

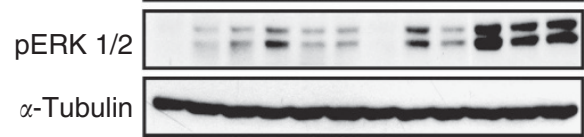

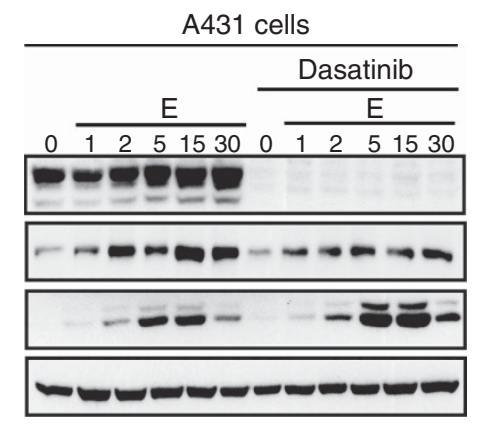

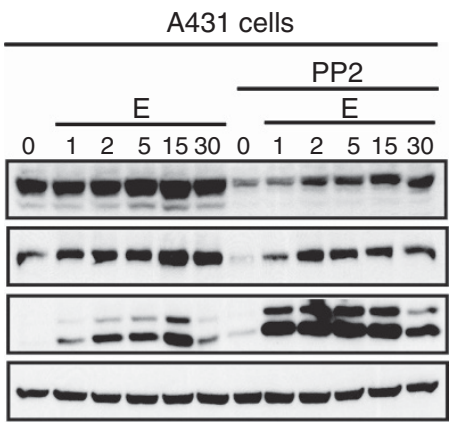

Figure 5. The SRC inhibitor PP2 recapitulated the effects of dasatinib on cell signalling. (A) Dasatinib and (B) PP2 drugs were used to treat FaDu and A431 cells. Cultures ( $\approx 12000$ cells per $\mathrm{cm}^{2}$ in $60 \mathrm{~mm}$ dishes) were incubated for 5 days and then treated in FBS-free medium with: (1) vehicles; (2) $150 \mathrm{~nm}$ dasatinib $\times 48 \mathrm{~h}$ or (3) $10 \mu \mathrm{M} \mathrm{PP} 2 \times 48 \mathrm{~h}$. Before cell lysis, cultures were stimulated with $10 \mathrm{ng} \mathrm{ml}^{-1}$ EGF ligand (E) $\times 1,2,5,15$ or $30 \mathrm{~min}$. $\alpha$-Tubulin was used as a loading control. $\alpha$-Tubulin, pSRC, pEGFR and pERK1/2 were analysed by western blot in whole lysates.

phosphorylated by SRC instead of by the EGFR itself, became inhibited by dasatinib. Specifically, dasatinib reduced the EGFinduced phosphorylation over a time course between 0 and $30 \mathrm{~min}$ in A431 cells and between 15 and 30 min in FaDu cells (Figure 5A). Phosphorylation of site Y845 is a potential mechanism to bypass EGFR blockage and resistance to anti-EGFR therapies, and according to this finding dasatinib would be useful to overcome this type of resistance. However, the inhibition of phosphorylation of EGFR-Y845 by dasatinib was not reflected downstream of pERK1/2 levels. On the contrary, dasatinib induced a remarkable activation of ERK1/2, as was also observed previously in Figure 1B.
We know that dasatinib has inhibitory effects on Abelson tyrosine kinase (Abl), c-KIT kinase, the ephrin type-A receptor 2 (EPHA2) and platelet-derived growth factor $\beta$ receptor (PDGF $\beta$ ), among others (Lombardo et al, 2004). To test whether the effects of dasatinib could actually be attributed to SRC inhibition, we treated both types of cells with PP2 substance, a more specific inhibitor of SRC (Hanke et al, 1996). We found that PP2 inhibited the phosphorylation of SRC kinase at tyrosine residue Y418, inhibited the phosphorylation of EGFR Y845 and increased the levels of pERK1/2 in a consistent manner in both cell types, just as dasatinib did (Figure 5B). 
Dasatinib increases the activity of RAS in FaDu and A431 cells. We next attempted to delimit the position within the EGFR/ ERK1/2 pathway at which a stimulatory signal was generated by dasatinib. We hypothesised that the origin of the signal might be located at RAS protein or upstream of the RAS protein, a supposition supported by the fact that ERK1/2 and AKT share the protein RAS as a common upstream activator, and both oncoproteins were found induced by dasatinib in $\mathrm{FaDu}$ and A431 cells. We next found an increase in the levels of the active form of RAS molecule in the presence of dasatinib in A431 cells (Figure 6B). And more relevant, although treatment with cetuximab partially decreased RAS-GTP levels, the addition of dasatinib, rather than further inhibiting RAS, actually fuelled its activity in both cell lines (Figure 6A and B, lanes CE without dasatinib compared with lanes $\mathrm{CE}$ with dasatinib). These functional findings are consistent with our previous results showing an unexpected activation of AKT and ERK, and indicate that the origin of the antagonistic effect is located in an upstream position before the switch that activates RAS from its inactive GDP form to the GTP activated state.

\section{DISCUSSION}

In this study, we have focussed our interest on the paradoxical response of the $\mathrm{FaDu}$ and $\mathrm{A} 431$ cell lines to dasatinib and cetuximab rather than go into a deep evaluation of the SCC5, SCC25 and SCC29 cell lines that were sensitive to dasatinib. The main accomplishment of our study was to observe that anti-SRC therapy may counteract anti-EGFR therapies based on cetuximab

A

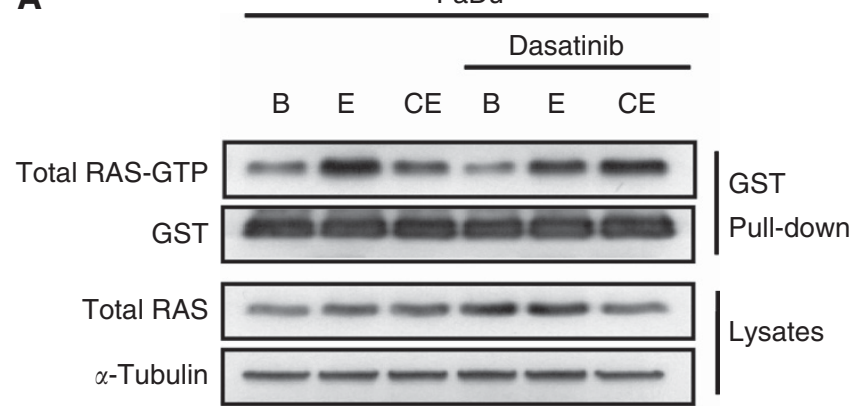

B

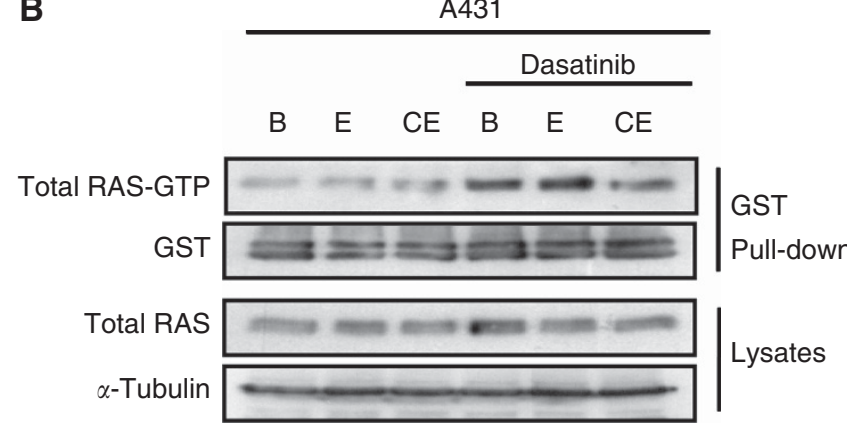

Figure 6. Effect of dasatinib on RAS activity in cell cultures treated with cetuximab. (A) The FaDu cells and (B) A431 cells ( $\approx 12000$ cells per $\mathrm{cm}^{2}$ in $60 \mathrm{~mm}$ dishes) were incubated for 5 days and then treated in FBS-free medium with: (1) vehicles (lane B) and (2) $30 \mathrm{~nm}$ cetuximab (lanes $C$ and $C E)$. Before cell lysis, cultures were stimulated with $10 \mathrm{ng} \mathrm{ml}^{-1} \mathrm{EGF}$ ligand $\times 10 \mathrm{~min}$ (lanes $\mathrm{E}$ and $\mathrm{CE}$ ). In parallel, a set of cultures was additionally treated with $150 \mathrm{~nm}$ dasatinib $\times 48 \mathrm{~h}$. The RAS-GTP and GST were analysed by western blot in GST pull-down supernatants, and $\alpha$-tubulin and RAS proteins in whole lysates. in some cell types, an interesting observation because a number of clinical trials are currently exploring this combination or the association of dasatinib with other therapies (Montero et al, 2011).

Several preclinical studies of dasatinib support clinical research to treat solid tumours in combination with approved therapies. In association with anti-EGFR family drugs, dasatinib has been shown to synergise HER-2/Neu-overexpressing breast cancer cells in combination with trastuzumab (Seoane et al, 2010), and K-RAS mutant CRC cells in combination with cetuximab (Dunn et al, 2011). Similarly, the SRC inhibitor AZD0530 also synergises the EGFR inhibitor gefitinib in cases of SCCHN with SRC overexpression (Koppikar et al, 2008). Anti-SRC drugs combined with radiotherapy have yielded interesting outcomes in preclinical investigations. Recent studies have shown that nuclear EGFR correlates with radiation resistance, indicating that nuclear EGFR is part of the DNA-damage repair complex (Dittmann et al, 2010; Toulany et al, 2011). Because the transport of EGFR is induced by ionising radiation (Dittmann et al, 2009), and this transport is dependent on SRC kinase, SRC has become a target to overcome oncogenesis, and resistance mediated by EGFR (Dittmann et al, 2008). In addition, SRC has been directly involved in DNA repair after radiotherapy in head and neck in xenografted tumours (Stegeman et al, 2013). In addition, yet other preclinical data suggest that dasatinib is a promising drug in combination with cetuximab because of the proven ability of dasatinib to resensitise cetuximab-resistant cancer cells by inhibiting nuclear EGFR shuttling (Li et al, 2009).

In our study, however, we show that dasatinib is directly involved in the loss of the antitumour effect of cetuximab in $\mathrm{FaDu}$ and A431 cells. Of note, we discovered this finding by using treatment schedules that attempted to reproduce the timing and doses used in the clinical setting. Accordingly, radiation was applied in daily multiple fractions and drugs were administered concurrently with radiotherapy. The increased tumour growth induced by dasatinib was associated with higher DNA synthesis and angiogenesis, thus suggesting that the origin of the counteracting effect of dasatinib relies on biochemical mechanisms rather than pharmacokinetic interactions. Moreover, we provide evidence that the inhibition of SRC may activate RAS, AKT and ERK1/2 in the cancer cells analysed. The ultimate mechanisms involved in this paradox are still uncertain; however, the increased growth of $\mathrm{FaDu}$ and A431 xenografts is conclusive.

To explain why dasatinib and PP2 induced the phosphorylation of AKT and ERK1/2, we speculate that a negative regulatory RAS protein or upstream to RAS protein (likely a phosphatase of EGFR) might be activated by SRC kinases. If true, SRC inhibition could prevent or alleviate a negative feedback loop to control the EFGR/ RAS pathway, resulting in an increased activity and the corresponding phosphorylation of the downstream kinases, AKT and ERK1/2. Although this mechanism could well be real, other mechanisms could certainly be involved in ERK activation in the presence of dasatinib or PP2. In NIH 3T3, SRC inhibition by PP2 appears to prevent RAF-1 negative regulation, thus leading to an increase of pERK1/2 levels in an RAS-independent manner (Lee et al, 2004). A recent article reported that dasatinib drives paradoxical activation of ERK1/2 in melanoma, pancreatic, NSCLC and CRC cell types with preserved RAS kinase activity. This appears to be because of partial inhibition of RAF kinases by dasatinib that facilitates the binding of BRAF to CRAF, resulting in a paradoxical CRAF hyperactivation and ERK1/2 phosphorylation (Packer et al, 2011). The essential role for RAS is to counteract RAF inhibition by binding to BRAF and CRAF to form dimers to eventually activate MEK/ERK kinases. The relevancy of this observation is that, in case of resistance to targeted therapy in which a certain amount of RAS activity is allowed, dasatinib would promote ERK activation. Interestingly, in our experimental model, cetuximab presented a partial inhibitory effect on RAS-GTP 
activity; thus, a loss of negative regulatory feedback loop on RAS or unexpected RAS-RAF interaction might have taken place resulting in paradoxical downstream signalling.

One noteworthy point is that the specific EGFR chemical inhibitor AG1478 appears to be extraordinarily effective on AKT and ERK1/2 in FaDu and A431 cells, both in the absence or presence of dasatinib. This result suggests that the function of EGFR is crucial for dasatinib-induced phosphorylation of AKT and ERK1/2. On the other hand, it implies that the ideal treatment for $\mathrm{FaDu}$ - and A431-derived tumours would have been AG1478 rather than cetuximab. AG1478 was able to block downstream signalling even in the presence of EGF and dasatinib, whereas the relatively cetuximab insensitive $\mathrm{FaDu}$ and $\mathrm{A} 431$ cells allowed EGFR signalling in response to EGF, a situation in which these cells could take profit when SRC is disabled. This would be a reactiveadaptive mechanism that some cells enable to survive when they are facing with biological cellular stress such as radiation and weak EGFR signalling, as we found in our experiments.

In conclusion, in this paper we demonstrate that adding dasatinib to radiotherapy combined with cetuximab to improve cancer control in FaDu- and A431-derived xenoimplanted tumours actually resulted in an antagonistic effect.

\section{ACKNOWLEDGEMENTS}

This work received financial support from Merck KgaA, Spanish Association Against Cancer, Barcelona Committee and AGAUR (SGR681) from the Catalan Government (Spain). We are grateful to $\mathrm{Mr}$ Bradley Londres for his excellent assistance in improving the English in the manuscript and to Dr Dani Azuara for helping with the mutational analysis of RAS.

\section{REFERENCES}

Amit I, Wides R, Yarden Y (2007) Evolvable signaling networks of receptor tyrosine kinases: relevance of robustness to malignancy and to cancer therapy. Mol Syst Biol 3: 151.

Baro M, de Llobet LI, Modolell I, Guedea F, Visa J, Balart J (2012) Development and refinement of a technique using a medical radiation therapy facility to irradiate immunodeficient mice bearing xenografted human tumours. Lab Anim 46: 345-348.

Bonner JA, Harari PM, Giralt J, Azarnia N, Shin DM, Cohen RB, Jones CU, Sur R, Raben D, Jassem J, Ove R, Kies MS, Baselga J, Youssoufian H, Amellal N, Rowinsky EK, Ang KK (2006) Radiotherapy plus cetuximab for squamous-cell carcinoma of the head and neck. N Engl J Med 354: 567-578.

Dittmann K, Mayer C, Fehrenbacher B, Schaller M, Kehlbach R, Rodemann HP (2010) Nuclear EGFR shuttling induced by ionizing radiation is regulated by phosphorylation at residue Thr654. FEBS Lett 584(18): 3878-3884.

Dittmann K, Mayer C, Fehrenbacher B, Schaller M, Raju U, Milas L, Chen DJ, Kehlbach R, Rodemann HP (2005) Radiation-induced epidermal growth factor receptor nuclear import is linked to activation of DNA-dependent protein kinase. J Biol Chem 280: 31182-31189.

Dittmann K, Mayer C, Kehlbach R, Rodemann HP (2008) Radiation-induced caveolin-1 associated EGFR internalization is linked with nuclear EGFR transport and activation of DNA-PK. Mol Cancer 7: 69.

Dittmann K, Mayer C, Kehlbach R, Rothmund MC, Peter Rodemann H (2009) Radiation-induced lipid peroxidation activates src kinase and triggers nuclear EGFR transport. Radiother Oncol 92: 379-382.

Dunn EF, Iida M, Myers RA, Campbell DA, Hintz KA, Armstrong EA, Li C, Wheeler DL (2011) Dasatinib sensitizes KRAS mutant colorectal tumors to cetuximab. Oncogene 30: 561-574.

Elsberger B, Stewart B, Tatarov O, Edwards J (2010) Is Src a viable target for treating solid tumours? Curr Cancer Drug Targets 10: 683-694.

Erjala K, Sundvall M, Junttila TT, Zhang N, Savisalo M, Mali P, Kulmala J, Pulkkinen J, Grenman R, Elenius K (2006) Signaling via ErbB2 and ErbB3 associates with resistance and epidermal growth factor receptor (EGFR) amplification with sensitivity to EGFR inhibitor gefitinib in head and neck squamous cell carcinoma cells. Clin Cancer Res 12: 4103-4111.
Giannoni E, Buricchi F, Raugei G, Ramponi G, Chiarugi P (2005) Intracellular reactive oxygen species activate Src tyrosine kinase during cell adhesion and anchorage-dependent cell growth. Mol Cell Biol 25: 6391-6403.

Hanahan D, Weinberg RA (2000) The hallmarks of cancer. Cell 100: 57-70. Hanahan D, Weinberg RA (2011) Hallmarks of cancer: the next generation. Cell 144: 646-674.

Hanke JH, Gardner JP, Dow RL, Changelian PS, Brissette WH, Weringer EJ, Pollok BA, Connelly PA (1996) Discovery of a novel, potent, and Src family-selective tyrosine kinase inhibitor. Study of Lck- and FynTdependent T cell activation. J Biol Chem 271: 695-701.

Hunter T (1997) Oncoprotein networks. Cell 88: 333-346.

Jorissen RN, Walker F, Pouliot N, Garrett TP, Ward CW, Burgess AW (2003) Epidermal growth factor receptor: mechanisms of activation and signalling. Exp Cell Res 284: 31-53.

Koppikar P, Choi SH, Egloff AM, Cai Q, Suzuki S, Freilino M, Nozawa H, Thomas SM, Gooding WE, Siegfried JM, Grandis JR (2008) Combined inhibition of c-Src and epidermal growth factor receptor abrogates growth and invasion of head and neck squamous cell carcinoma. Clin Cancer Res 14: 4284-4291.

Lee M, Kim JY, Anderson WB (2004) Src tyrosine kinase inhibitor PP2 markedly enhances Ras-independent activation of Raf-1 protein kinase by phorbol myristate acetate and $\mathrm{H} 2 \mathrm{O} 2$. J Biol Chem 279: 48692-48701.

Li C, Iida M, Dunn EF, Ghia AJ, Wheeler DL (2009) Nuclear EGFR contributes to acquired resistance to cetuximab. Oncogene 28: 3801-3813.

Liang K, Ang KK, Milas L, Hunter N, Fan Z (2003) The epidermal growth factor receptor mediates radioresistance. Int J Radiat Oncol Biol Phys 57: 246-254.

Lin SY, Makino K, Xia W, Matin A, Wen Y, Kwong KY, Bourguignon L, Hung MC (2001) Nuclear localization of EGF receptor and its potential new role as a transcription factor. Nat Cell Biol 3: 802-808.

Lin YC, Wu MH, Wei TT, Chuang SH, Chen KF, Cheng AL, Chen CC (2012) Degradation of epidermal growth factor receptor mediates dasatinibinduced apoptosis in head and neck squamous cell carcinoma cells. Neoplasia 14: 463-475.

Lombardo LJ, Lee FY, Chen P, Norris D, Barrish JC, Behnia K, Castaneda S, Cornelius LA, Das J, Doweyko AM, Fairchild C, Hunt JT, Inigo I, Johnston K, Kamath A, Kan D, Klei H, Marathe P, Pang S, Peterson R, Pitt S, Schieven GL, Schmidt RJ, Tokarski J, Wen ML, Wityak J, Borzilleri RM (2004) Discovery of N-(2-chloro-6-methyl- phenyl)-2-(6-(4-(2hydroxyethyl)- piperazin-1-yl)-2-methylpyrimidin-4- ylamino)thiazole-5carboxamide (BMS-354825), a dual Src/Abl kinase inhibitor with potent antitumor activity in preclinical assays. J Med Chem 47: 6658-6661.

Luo FR, Barrett YC, Yang Z, Camuso A, McGlinchey K, Wen ML, Smykla R, Fager K, Wild R, Palme H, Galbraith S, Blackwood-Chirchir A, Lee FY (2008) Identification and validation of phospho-SRC, a novel and potential pharmacodynamic biomarker for dasatinib (SPRYCEL), a multitargeted kinase inhibitor. Cancer Chemother Pharmacol 62: 1065-1074.

Malumbres M, Barbacid M (2003) RAS oncogenes: the first 30 years. Nat Rev Cancer 3: 459-465.

Montero JC, Seoane S, Ocana A, Pandiella A (2011) Inhibition of SRC family kinases and receptor tyrosine kinases by dasatinib: possible combinations in solid tumors. Clin Cancer Res 17: 5546-5552.

Nowsheen S, Bonner JA, Yang ES (2011) The poly(ADP-Ribose) polymerase inhibitor ABT-888 reduces radiation-induced nuclear EGFR and augments head and neck tumor response to radiotherapy. Radiother Oncol 99: 331-338.

Packer LM, Rana S, Hayward R, O'Hare T, Eide CA, Rebocho A, Heidorn S, Zabriskie MS, Niculescu-Duvaz I, Druker BJ, Springer C, Marais R (2011) Nilotinib and MEK inhibitors induce synthetic lethality through paradoxical activation of RAF in drug-resistant chronic myeloid leukemia. Cancer Cell 20: 715-727.

Pueyo G, Mesia R, Figueras A, Lozano A, Baro M, Vazquez S, Capella G, Balart J (2010) Cetuximab may inhibit tumor growth and angiogenesis induced by ionizing radiation: a preclinical rationale for maintenance treatment after radiotherapy. Oncologist 15: 976-986.

Raben D, Helfrich B, Chan DC, Ciardiello F, Zhao L, Franklin W, Barón AE, Zeng C, Johnson TK, Bunn Jr PA (2005) The effects of cetuximab alone and in combination with radiation and/or chemotherapy in lung cancer. Clin Cancer Res 11: 795-805.

Roskoski Jr R (2004) Src protein-tyrosine kinase structure and regulation. Biochem Biophys Res Commun 324: 1155-1164. 
Sato K, Nagao T, Iwasaki T, Nishihira Y, Fukami Y (2003) Src-dependent phosphorylation of the EGF receptor Tyr-845 mediates Stat-p21waf1 pathway in A431 cells. Genes Cells 8: 995-1003.

Schmidt-Ullrich RK, Mikkelsen RB, Dent P, Todd DG, Valerie K, Kavanagh BD, Contessa JN, Rorrer WK, Chen PB (1997) Radiationinduced proliferation of the human A431 squamous carcinoma cells is dependent on EGFR tyrosine phosphorylation. Oncogene 15: 1191-1197.

Seoane S, Montero JC, Ocana A, Pandiella A (2010) Effect of multikinase inhibitors on caspase-independent cell death and DNA damage in HER2-overexpressing breast cancer cells. J Natl Cancer Inst 102: 1432-1446.

Song JY, Lee SW, Hong JP, Chang SE, Choe H, Choi J (2009) Epidermal growth factor competes with EGF receptor inhibitors to induce cell death in EGFR-overexpressing tumor cells. Cancer Lett 283: 135-142.

Sordella R, Bell DW, Haber DA, Settleman J (2004) Gefitinib-sensitizing EGFR mutations in lung cancer activate anti-apoptotic pathways. Science 305: 1163-1167.

Stegeman H, Span PN, Rijken PF, Cockx SC, Wheeler DL, Iida M, van der Kogel AJ, Kaanders JH, Bussink J (2013) Dasatinib inhibits DNA repair after radiotherapy specifically in pSFK-expressing tumor areas in head and neck xenograft tumors. Transl Oncol 6: 413-419.

Summy JM, Gallick GE (2006) Treatment for advanced tumors: SRC reclaims center stage. Clin Cancer Res 12: 1398-1401.

Tice DA, Biscardi JS, Nickles AL, Parsons SJ (1999) Mechanism of biological synergy between cellular Src and epidermal growth factor receptor. Proc Natl Acad Sci USA 96: 1415-1420.
Toulany M, Schickfluss TA, Fattah KR, Lee KJ, Chen BP, Fehrenbacher B, Schaller M, Chen DJ, Rodemann HP (2011) Function of erbB receptors and DNA-PKcs on phosphorylation of cytoplasmic and nuclear Akt at S473 induced by erbB1 ligand and ionizing radiation. Radiother Oncol 101: $140-146$

Weidner N, Semple JP, Welch WR, Folkman J (1991) Tumor angiogenesis and metastasis-correlation in invasive breast carcinoma. N Engl J Med 324: $1-8$.

Workman P, Aboagye EO, Balkwill F, Balmain A, Bruder G, Chaplin DJ, Double JA, Everitt J, Farningham DA, Glennie MJ, Kelland LR, Robinson V, Stratford IJ, Tozer GM, Watson S, Wedge SR, Eccles SA (2010) Guidelines for the welfare and use of animals in cancer research. Br J Cancer 102: 1555-1577.

Wu Z, Doondeea JB, Gholami AM, Janning MC, Lemeer S, Kramer K, Eccles SA, Gollin SM, Grenman R, Walch A, Feller SM, Kuster B (2011) Quantitative chemical proteomics reveals new potential drug targets in head and neck cancer. Mol Cell Proteomics 10: M111 011635.

Wyckoff J, Wang W, Lin EY, Wang Y, Pixley F, Stanley ER, Graf T, Pollard JW, Segall J, Condeelis J (2004) A paracrine loop between tumor cells and macrophages is required for tumor cell migration in mammary tumors. Cancer Res 64: 7022-7029.

This work is published under the standard license to publish agreement. After 12 months the work will become freely available and the license terms will switch to a Creative Commons AttributionNonCommercial-Share Alike 3.0 Unported License.

Supplementary Information accompanies this paper on British Journal of Cancer website (http://www.nature.com/bjc) 